\title{
Subtraction Magnetocardiogram for Detecting Coronary Heart Disease
}

\author{
Akihiko Kandori, Ph.D., ${ }^{*}$ Kuniomi Ogata, ${ }^{*}$ Tsuyoshi Miyashita, M.S., ${ }^{*}$ \\ Hiroshi Takaki, Ph.D., M.D., † Hideyuki Kanzaki, Ph.D., M.D., $†$ Syuji Hashimoto, $\uparrow$ \\ Wataru Shimizu, Ph.D., M.D., † Shiro Kamakura, Ph.D., M.D., $†$ \\ Shigeyuki Watanabe, Ph.D., M.D., $\ddagger$ and Kazutaka Aonuma, Ph.D. $\ddagger$ \\ From the *Advanced Research Laboratory, Kokubunji, Tokyo, Japan; †National Cardiovascular Center, Osaka, \\ Japan; and $\ddagger$ University of Tsukuba, Ibaraki, Japan
}

Background: A large-scale magnetocardiogram (MCG) database was produced, and standard MCG waveforms of healthy patients were calculated by using this database. It was clarified that the standard MCG waveforms are formed with the same shape and current distribution in healthy patients. A new subtraction method for detecting abnormal ST-T waveforms in coronary heart disease (CHD) patients by using the standard MCG waveform was developed.

Methods: We used MCGs of 56 CHD patients (63 \pm 3 years old) and 101 age-matched normal control patients $(65 \pm 5$ years old). To construct a subtracted ST-T waveform, we used standard MCG waveforms produced from 464 normal MCGs (male: 268, female: 196). The standard MCG waveforms were subtracted from each subject's measured MCGs, which were shortened or lengthened and normalized to adjust to the data length and magnitude of the standard waveform. We evaluated the maximum amplitude and maximum current-arrow magnitude of the subtracted ST-T waveform.

Results: The maximum magnetic field, maximum magnitude of current arrows, and maximum magnitude of total current vector increased according to the number of coronary artery lesions. The sensitivity and specificity of detecting CHD and normal control patients were $74.6 \%$ and $84.1 \%$, respectively.

Conclusions: The subtraction MCG method can be used to detect CHD with high accuracy, namely, sensitivity of $74.6 \%$ and specificity of $84.1 \%$ (in the case of maximum amplitude of total current vector). Furthermore, the subtraction MCG magnitude and its current distribution can reflect the expanse of the ischemic lesion area and the progress from ischemia to myocardial infarction.

Ann Noninvasive Electrocardiol 2010;15(4):360-368

magnetocardiogram; subtraction; current-arrow map; Coronary Heart Disease

\section{INTRODUCTION}

Coronary heart disease (CHD) is the most common cause of death in the world. Advances in noninvasive coronary-artery imaging, such as $\mathrm{x}$ ray coronary angiography ${ }^{1}$ and multidetector computed tomography $(\mathrm{CT}){ }_{1,2}^{1,2}$ has enabled early and precise detection of CHD. These tools are needed to treat artery constriction and improve the symptoms of angina. On the other hand, as functional tests of myocardial activity, exercise electrocardiogram (ECG) and single-photon-emission computed tomography (SPECT) are widely used for detecting signs of cardiac sudden death in CHD patients., However, the distribution of myocardial electrical currents, which include information on damaged tissues, cannot be detected with these cardiac tests.

Noncontact magnetocardiograms (MCGs) can produce a myocardial-current-distribution map by detecting weak magnetic fields generated by myocardial electrical currents. The morphology of MCG waveforms produced from electrical currents is similar for each person because the MCG signals are less affected by organ conductivity, and the

Address for correspondence: Akihiko Kandori, Advanced Research Laboratory, Hitachi Ltd., 1-280 Higashi-Koigakubo, Kokubunji, Tokyo 185-8601, Japan. Fax: +81-42-327-7783; E-mail: akihiko.kandori.vc@hitachi.com 
two-dimensional currents in the heart only produce a magnetic field. Given those facts, we constructed a database of 464 adult healthy patients ${ }^{5}$ and a template MCG waveform. ${ }^{6}$ In particular, the database and template enable the normal activity in the ST-T wave to be subtracted because of calm repolarization. Furthermore, some researchers have reported high CHD detection sensitivity ${ }^{7-15}$ when MCGs are used because the ST-T MCG waves of CHD patients have an abnormal pattern. And MCGs are useful in detecting myocardial infarction (MI) in non-ST-segment elevation. ${ }^{14,15}$

To detect ST-T abnormality in CHD patients, we used our MCG-waveform template to construct a subtraction ST-T waveform. Subtraction of ST-T waveforms of CHD patients and control patients is used to evaluate the efficacy of detecting CHD, the difference between electrical current abnormalities in lesion areas, and the difference between ischemia and MI.

\section{METHODS \\ Participants}

Fifty-six Japanese CHD patients (10 females and 46 males) and 101 Japanese normal control patients (31 females and 70 males) participated, as listed in Table 1. This population is the same as that of our previous study, ${ }^{16}$ which clarified CHD patients' spatial current abnormalities defined as a vessellesion diameter of $>75 \%$. The CHD patientswith two or three coronary-vessel lesions were defined as those with two lesions or three vessel lesions with diameters of $\geq 75 \%$. We obtained informed consent from all participants and received approval from relevant ethical committees. Moreover, the CHD patients were classified into two groups, namely, ischemic $(\mathrm{n}=12)$ or $\mathrm{MI}(\mathrm{n}=12)$, to evaluate the detection performance for ischemia, and patients with both ischemic and infarct areas were excluded. The ischemia and MI patients could be categorized as a minor part of the CHD patients because these patients were examined by SPECT.

\section{MCG Recordings and Setup}

MCG recording was performed above the chest of each participant for 30 seconds. The MCG signals were detected with a 64-channel MCG system (Hitachi High-Technologies Ltd., Tokyo, Japan) ${ }^{17}$ in a magnetically shielded room. The detected
Table 1. Numbers of CHD and Control Patients

\begin{tabular}{|c|c|c|}
\hline & $N(n=101)$ & CHD $(n=56)$ \\
\hline $\begin{array}{l}\text { Age (years) } \\
\text { Gender }\end{array}$ & $63 \pm 3$ & $65 \pm 6$ \\
\hline Female/Male & $31 / 70$ & $10 / 46$ \\
\hline BMI & 23.7 & 24.0 \\
\hline $\mathrm{HR}$ & 63 & 63 \\
\hline SVD & & 25 \\
\hline LAD & & 16 (P:6, M: 5, D: 0, U: 5) \\
\hline LCX & & $3(\mathrm{P}: 1, \mathrm{M}: 0, \mathrm{D}: 1, \mathrm{U}: 1)$ \\
\hline RCA & & $6(P: 1, M: 2, D: 2, U: 1)$ \\
\hline \multirow[t]{3}{*}{ DVD } & & 12 \\
\hline & & LAD + LCX: 6 \\
\hline & & LAD + RCA: 4 \\
\hline TVD & & $\begin{array}{c}\text { LCA + KLA: } 2 \\
19\end{array}$ \\
\hline
\end{tabular}

$\mathrm{CHD}=$ coronary heart disease, coronary artery lesion $(>$ 75\%); $\mathrm{D}=$ distal coronary artery; DVD = double vessel disease; $\mathrm{LAD}=$ left anterior descending coronary artery; LCX = left circumflex coronary artery; $\mathrm{M}=$ middle coronary artery; $\mathrm{N}=$ age-matched normal controls with no history of cardiovascular disease and normal electrocardiogram (ECG) at rest; $\mathrm{P}=$ proximal coronary artery; $\mathrm{RCA}=$ right coronary artery; SVD = single vessel disease; TVD = triple vessel disease; $U=$ unknown precise location.

MCG signals were passed through an analog bandpass filter $(0.1-100 \mathrm{~Hz})$ and an analog notch filter $(50 \mathrm{~Hz})$. The signals were then digitized at a sampling rate of $1 \mathrm{kHz}$ by using an analog-digital converter. To remove the noise in the signals, the MCG data were averaged 20-30 times by using an ECG signal trigger. The MCG data were measured at three hospitals (Hitachi General Hospital, the National Cardiovascular Center, and University of Tsukuba Hospital) by using the same MCG system with the same sensitivity.

\section{Production of Subtracted MCG Waveforms}

We focused on the ST-T segment in the MCG waveforms because previous studies reported that electrical current abnormality in CHD patients appears during ventricular repolarization in MCGs. ${ }^{7-15}$ The $\mathrm{T}$ end of each patient was manually defined by using overlapped MCG waveforms. ${ }^{5}$

A subtracted ST-T waveform was calculated by subtracting the standard MCG waveform, which was produced by averaging the 464 normal control patients' MCGs, ${ }^{6}$ from the measured MCG waveforms on each channel. Figure 1 shows the procedure for subtracting the ST-T waveforms. The time 


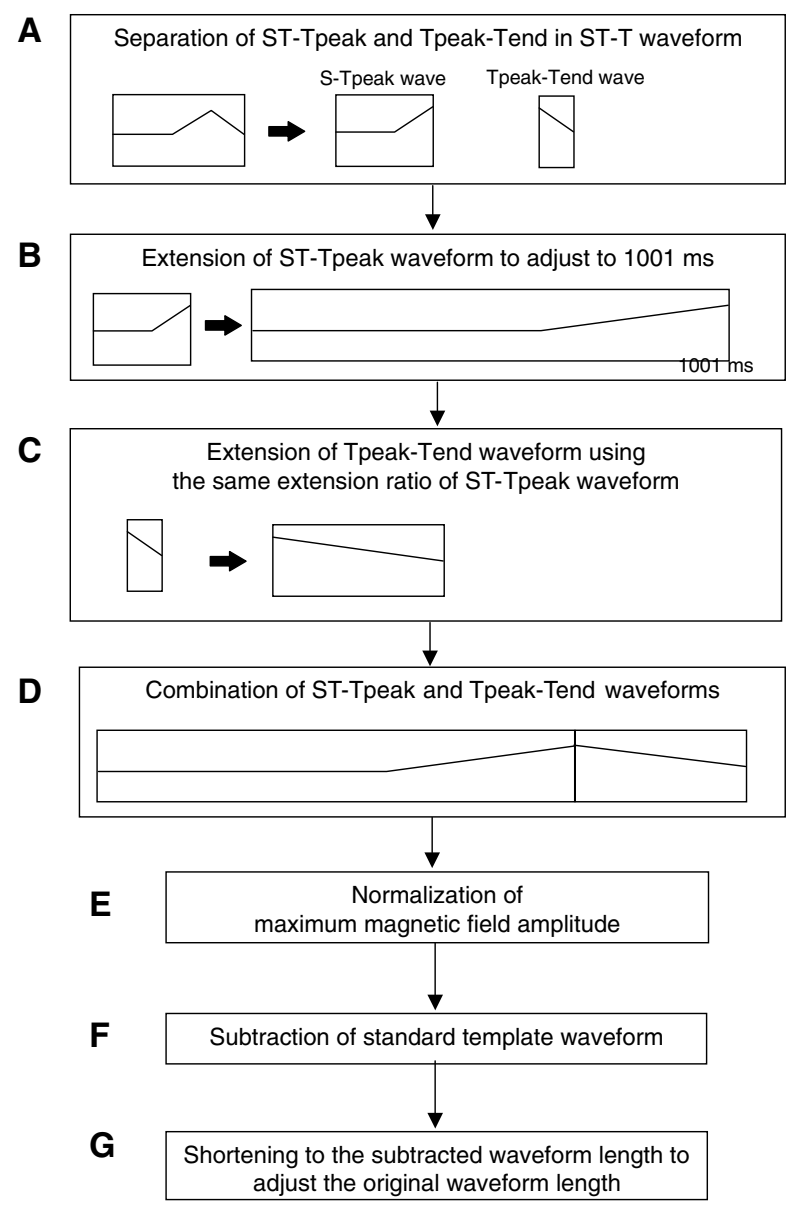

Figure 1. Procedure of subtracting ST-T waveform.

at which the amplitude of the ST-T waveform is a maximum varied among participants. The ST-T waveforms are, therefore, first separated into two parts, namely, an ST-Tpeak and a Tpeak-Tend, as shown in Figure 1A. Second, the ST-Tpeak waveforms are extended to 1001 milliseconds (Fig. 1B) by using the ratio of the duration of the ST-Tpeak waveform to the extended waveform. Third, the Tpeak-Tend waveforms are extended to 1001 milliseconds by using the same ratio for each participant (Fig. 1C).

In the next step, the ST-Tpeak and Tpeak-Tend waveforms are combined (Fig. 1D). The time interval of the data is lengthened by adding data points with zero amplitude after the Tend, and the amplitude of the magnetic field of both the Tpeak and Tpeak-Tend waveforms is normalized (Fig. 1E). The standard template waveform is then subtracted from the normalized ST-T waveforms
(Fig. 1F). Finally, the time interval of the subtracted ST-T-waveforms is shortened to fit the original time interval (Fig. 1G). The subtracted ST-T MCG waveforms for each participant were obtained by this procedure.

\section{Evaluation Method for Subtracting Waveforms}

MCG parameters, which can be produced from the amplitude of the magnetic field and current distribution, are used to quantitatively evaluate the residual MCG signals in the subtracted ST-T waveforms. To investigate the current distribution, a current-arrow map (CAM) is used to indicate current vectors in the heart. The CAM indicates pseudo currents (Ix and Iy) defined by the derivatives of the normal component $(\mathrm{Bz})$ of the MCG signals as

$I_{x, n}=\frac{d B_{z, n}}{d y}$

and

$I_{y, n}=-\frac{d B_{z, n}}{d x}$.

The magnitude of the current arrows $\left(I_{x, y}=\right.$ $\left.\sqrt{\left(I_{x, n}\right)^{2}+\left(I_{y, n}\right)^{2}}\right)$ is plotted as a contour map, where $n$ indicates channel number. The CAM helps in interpreting spatial heart electrical activity, and it can visualize the residual current component.

The above-mentioned CAM and the amplitude of the magnetic field at the Tpeak are used to calculate the following five parameters (three magnitude parameters and two two-dimensional distribution parameters).

1. Absolute value of the maximum amplitude of all MCG signals.

2. Absolute value of the maximum vector amplitude of all current vectors.

3. Maximum amplitude of total current vector (TCV).

The $\mathrm{TCV}^{5,18}$ is obtained by summing all current arrows. The TCV $(\mathbf{I})$ of all current arrows can be calculated as a vector value by using the current arrow $\left(\mathbf{I}_{\mathbf{n}}\right)$ at each sensor,

$\mathrm{I}=\sum_{n=1}^{64} \mathrm{I}_{\mathrm{n}}(T)$ 


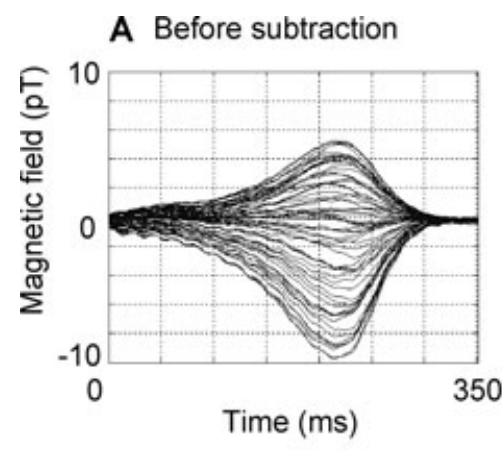

C CAM of subtracted waveform
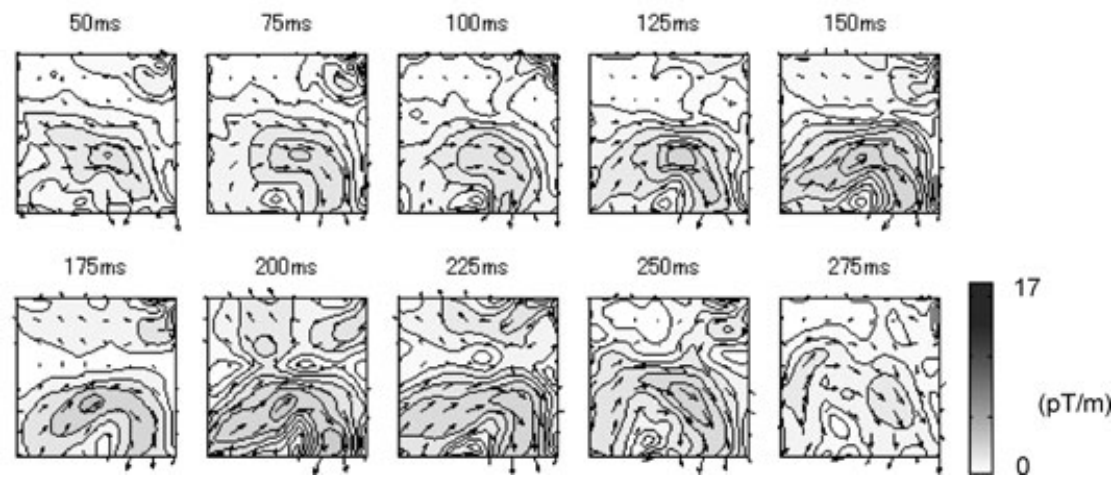

Figure 2. Subtracted ST-T waveform and current-arrow map (CAM) of typical normal control. (A) Original ST-T waveform. (B) Subtracted ST-T waveform. (C) CAM of (B).

where $T$ is the calculated time. The TCV amplitude of the subtracted waveform is used because TCV has high sensitivity for detecting CHD.

4. Area ratio of abnormal current vector appearance.

Average (A) and standard deviation $(\sigma)$ of the maximum current vector derived from step 2 in all healthy control patients were calculated in order to evaluate the abnormal current area. The number of current vectors with abnormal values above $\mathrm{A}+\sigma$ is counted, and the number of abnormal current vectors is divided by total channel number, 64 . The result of this division (given as a percentage) is used as the ratio of the areas where abnormal current vectors appear in the participant and control patients, hereafter referred to as the "area ratio of abnormal-current-vector appearance." For example, $100 \%$ indicates that all channels have abnormal values $(>\mathrm{A}+\sigma)$, and $25 \%$ indicates that 16 channels have abnormal values $(>\mathrm{A}+\sigma)$.
5. Two-dimensional frequency distribution of abnormal-current appearance

In the same manner as step 4, the number of appearances of abnormal-current vectors above $\mathrm{A}+$ $\sigma$ on each channel for each lesion group of CHD patients count is counted. The number (given as a percentage) is divided by the total number of participants in each lesion group. The number of appearances is plotted as a two-dimensional contour map of cumulative frequency. For instance, the $100 \%$ position in the two-dimensional contour map of three-vessel stenosis indicates that the $100 \%$ position always has an abnormal value $(>\mathrm{A}+\sigma)$. In this study, a two-dimensional contour map of each lesion group of the coronary artery was drawn.

\section{RESULTS}

\section{Typical Subtracted ST-T waveform}

A typical subtracted ST-T waveform and CAM of a healthy control is shown in Figure 2. While 


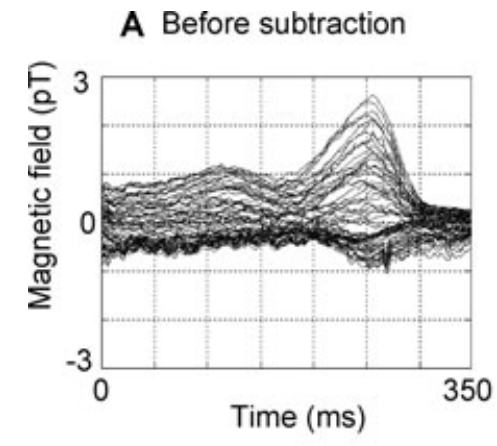

C CAM of subtracted waveform
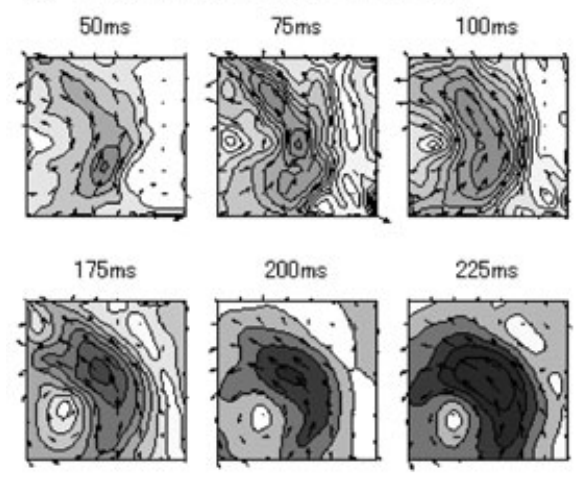

B After subtraction
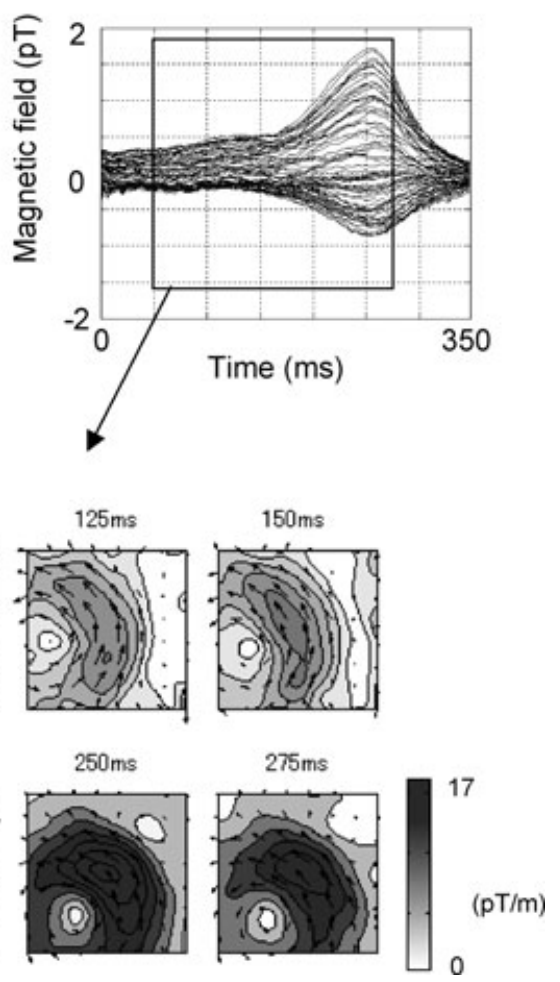

Figure 3. Subtracted ST-T waveform and current-arrow map (CAM) of typical CHD patient with triple vessel lesion. (A) Original ST-T waveform. (B) Subtracted ST-T waveform. (C) CAM of (B).

the amplitude of the ST-T waveform ranges from about -10 to $5 \mathrm{pT}$ (Fig. 2A) before subtraction, the residual amplitude is very small (Fig. 2B) after subtraction, ranging from about -0.02 to 0.01 pT. In this case, up to $99.8 \%$ of the ST-T waveform can be removed. In the CAM in Figure 2C, the current vectors have small amplitude, and the residual currents are weaker than those before subtraction.

Figure 3 shows a typical subtracted ST-T waveform and the CAM of triple vessel disease (TVD) in CHD patients. The amplitude of the ST-T waveform ranges from about -1 to $2.5 \mathrm{pT}$ (Fig. 3A) before the subtraction procedure, and the amplitude after the procedure is still high, ranging from about -0.9 to $1.8 \mathrm{pT}$. The reduction rate is about $23 \%$. In the CAM in Figure 3C, a right upward current with high amplitude (about 24 $\mathrm{pT} / \mathrm{m}$ ) appears. This pattern, with large residual ST-T components, is found in almost all CHD patients.

\section{Relationship between Parameters and Coronary-Artery Lesions}

Figure 4 shows the maximum amplitude of the magnetic field, maximum current vector, maximum amplitude of the TCV, and area ratio of abnormal current vector appearance, which were calculated from the subtracted ST-T waveform and its CAM of the control and CHD patients. In the case of all parameters, the mean values increase with increasing number of coronary artery lesions. The difference between control and lesion parameters is significant $(\mathrm{P}<0.01)$.

The sensitivity and specificity of the MCG for detecting CHD by using these four MCG parameters, listed in Table 2, were calculated. Criteria for each parameter are defined as the mean value plus the standard deviation for healthy control patients. In Table 2, the maximum amplitude of the TCV is highly sensitive (i.e., 74\%) and has specificity of $84 \%$, and the area ratio of abnormal current vector appearance has the highest specificity of $92.2 \%$. 


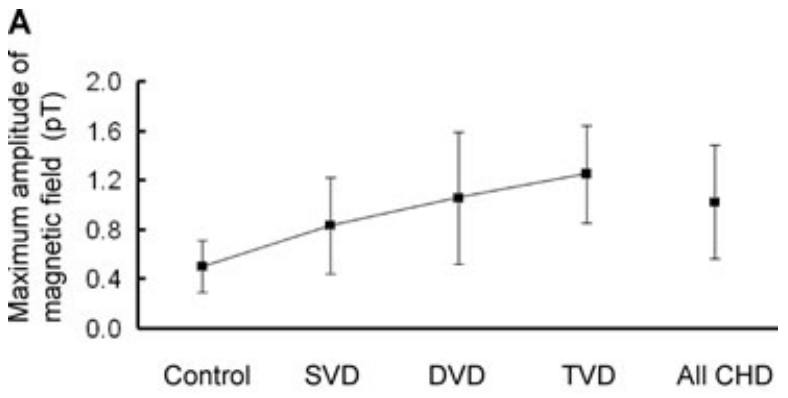

B
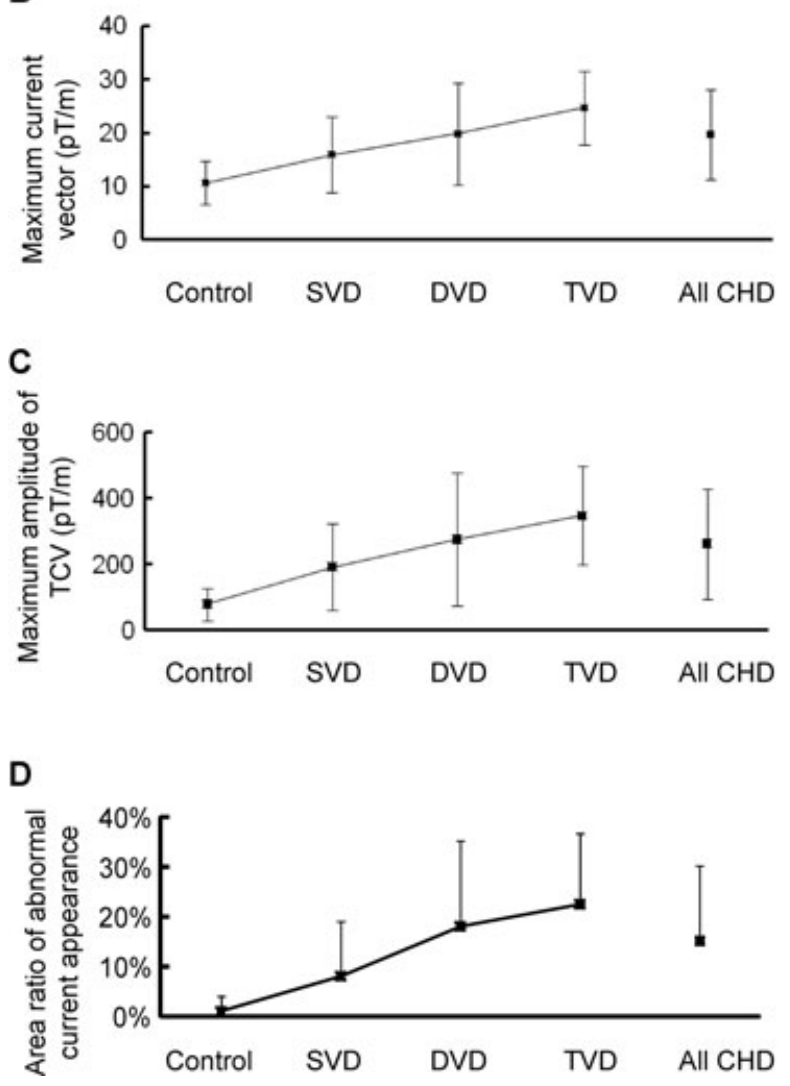

Figure 4. Relationship between each parameter and each lesion. (A) Maximum amplitude of magnetic field, (B) maximum current vector, (C) maximum amplitude of TCV, (D) area ratio of abnormal current vector appearance.

Two-dimensional frequency distribution of abnormal current vector appearance in each lesion patient is summarized in Figure 5. In the healthy control patients, there are no abnormalities in all measurement areas. In the left-anteriordescending (LAD) coronary artery of single-vesseldisease (SVD) patients, there is a $30 \%$ probability of abnormal current vector appearance near the septum and the left ventricular anterior wall. In the
Table 2. Summarized List of Sensitivity and Specificity of Each Parameter

\begin{tabular}{lcc}
\hline & $\begin{array}{c}\text { Sensitivity } \\
\text { (\%) }\end{array}$ & $\begin{array}{c}\text { Specificity } \\
\text { (\%) }\end{array}$ \\
\hline Maximum amplitude of & 67.2 & 86 \\
$\quad$ magnetic field & 67.3 & 84.5 \\
Maximum current vector & 74.6 & 84.1 \\
Maximum amplitude of TCV & 60.0 & 92.2 \\
Area ratio of abnormal & & \\
$\quad$ current vector & & \\
$\quad$ appearance & & \\
\hline
\end{tabular}

$\mathrm{TCV}=$ total current vector

left-circumflex (LCX) coronary artery of SVD patients, there is a $50 \%$ probability throughout the left and right ventricular walls. In the right coronary artery (RCA) of SVD patients, there is a $30 \%$ probability near the septum and right ventricular wall. The $30 \%$ probability in the LAD and LCX coronary arteries of double-vessel-disease (DVD) patients is similar to that in the SVD-LAD artery, and the probability remains the same throughout the right ventricular wall and apex. In the LAD and RCA coronary arteries of DVD patients, there is $50 \%$ probability throughout the left ventricle wall to the septum and the top of the right ventricle. In the LCX and RCA coronary arteries of DVD patients, there is a high probability $(90 \%)$ from the left ventricle upward. In patients with TVD, there is a $60 \%$ probability throughout the left and right ventricle walls.

\section{Relationship between MI and Ischemic Groups}

The four MCG parameters (maximum amplitude of magnetic field, maximum current vector, maximum amplitude of TCV, and area ratio of abnormal current vector appearance) for the MI and ischemic groups are plotted in Figure 6. These groups have much higher values for all parameters, and they significantly differ from those of the control patients $(\mathrm{P}<0.01)$. Moreover, the values of all parameters of the MI group are higher than those of the ischemic group.

\section{DISCUSSION}

\section{Advantages of the Subtraction Method}

As for the subtraction method, the ST-T waveform is separated into two segments, namely, an 


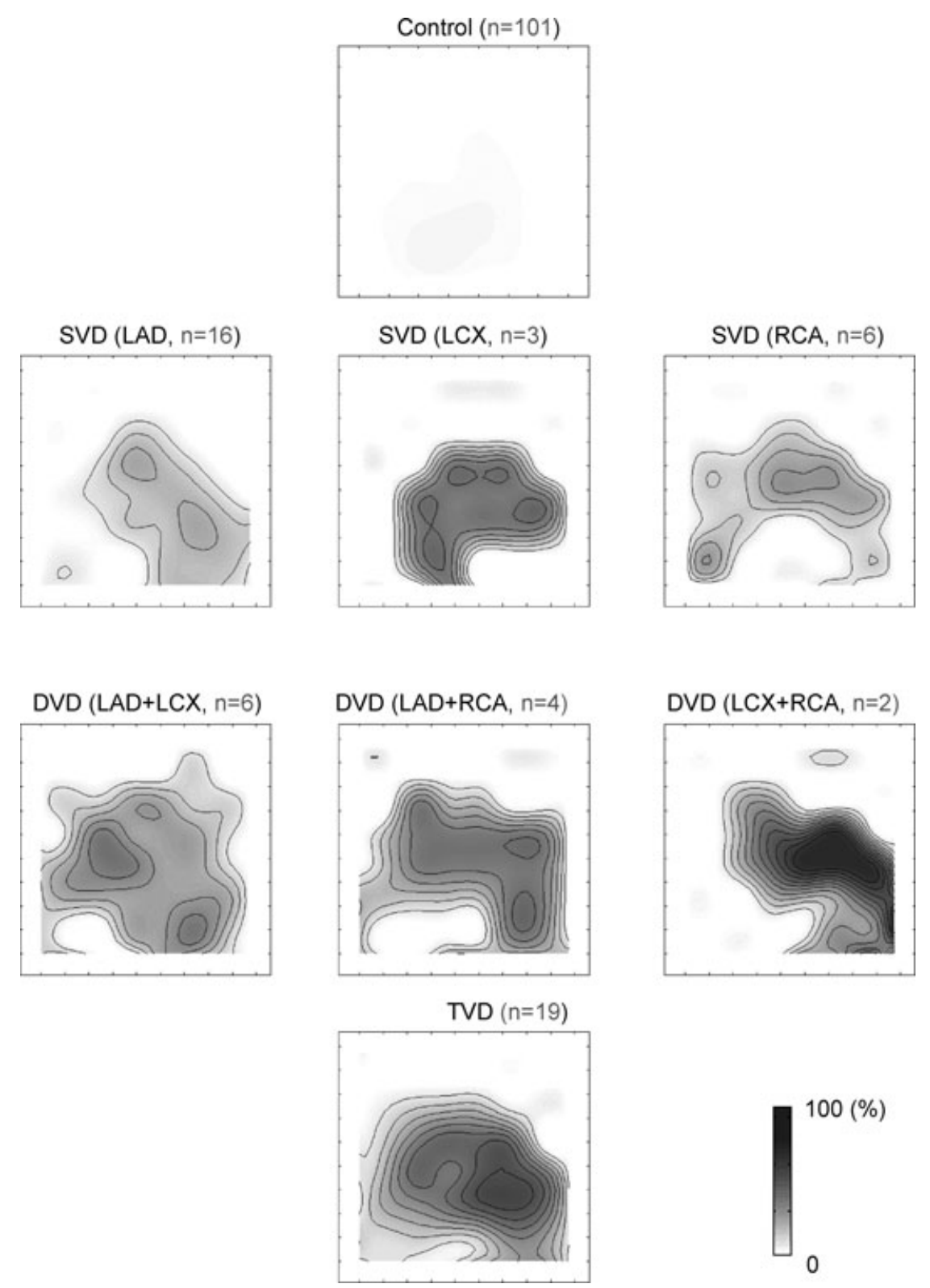

Figure 5. Two-dimensional frequency distribution of abnormal current appearance in each lesion patient and control.

ST-Tpeak and a Tpeak-Tend. To calibrate the Tpeak time for all subjects, the separation was necessary because the Tpeak time varied for each subject. If the Tpeak time is different for each subject, the template ST-T waveform (which is used in the subtraction method) will be an incomplete shape because the amplitude of the produced ST$\mathrm{T}$ waveform is very small (due to the waveformaveraging process). The difference between the Tpeak time for each subject may be associated with the difference in the action potential duration of the epicardium of each patient. ${ }^{19}$ The waveform separation, therefore, compensates the individual differences of Tpeak time, in contrast with the general averaging method for removing external signals such as maternal signals ${ }^{20}$ and ventricular activity. ${ }^{21}$

After the separation, the ST-Tpeak segment was extended to 1001 milliseconds $(1000 \pm 1$ milliseconds) to normalize the time duration. The last 


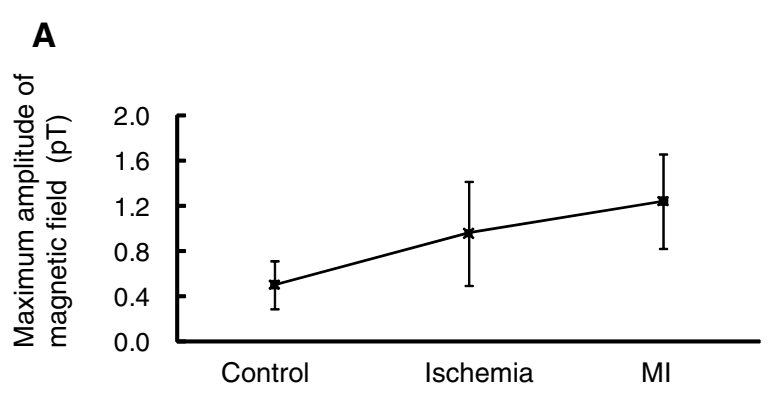

B

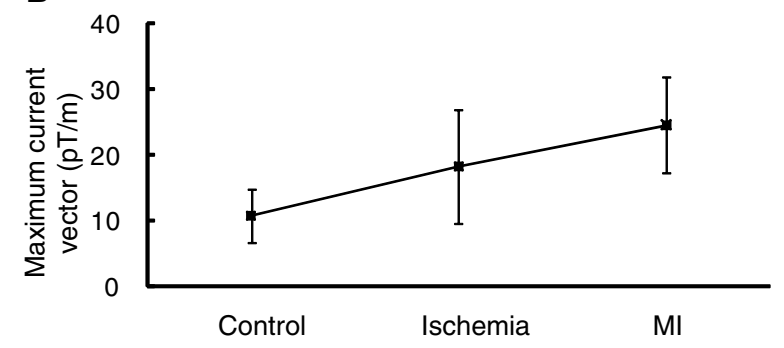

C

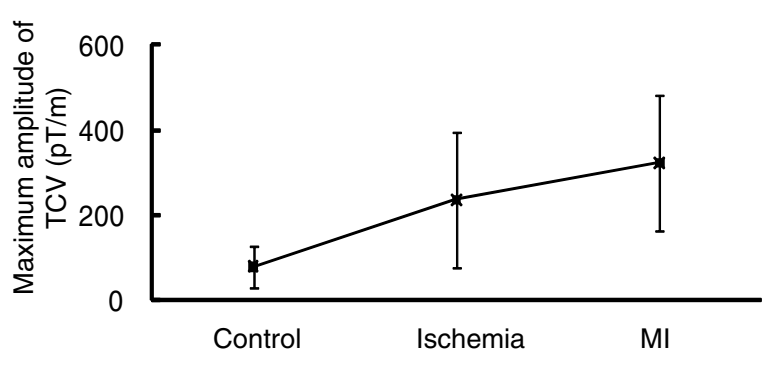

D

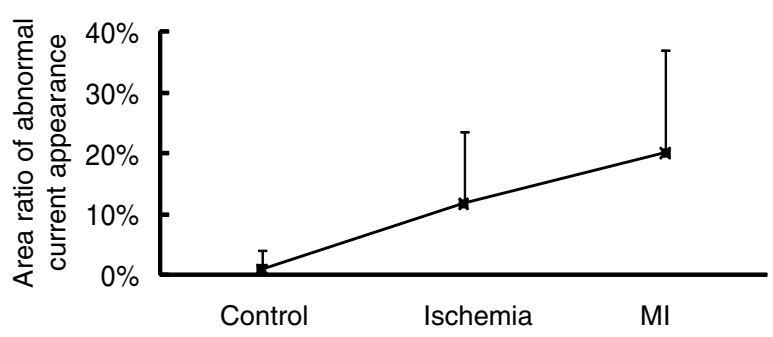

Figure 6. Relationships among control, ischemia, and myocardial infarction. (A) Maximum amplitude of magnetic field, (B) maximum current vector, (C) maximum amplitude of total current vector, (D) area ratio of abnormal current vector appearance.

time point, namely, the last 1 milliseconds in 1001 milliseconds, was used to superpose the normalized Tpeak-Tend waveform on the normalized STTpeak waveform. On the other hand, 1000 milliseconds was chosen for easy calculation of the normalization ratio, and 1000 milliseconds must be sufficiently longer than a general ST-Tend time in order to obtain a positive normalization ratio. The authors, therefore, consider that 1000 milliseconds is adequate time for making the template waveform.

The subtracted ST-T peak of normal subjects is significantly decreased in Figure 2B, while that of a CHD patient (Fig. 3B) has a higher amplitude. The difference between the peaks indicates that a normal electrical-current vector with the same direction exists in the ventricular area. It is, therefore, considered that the subtracted waveforms of the CHD patients are produced by the abnormal residual currents (which indicate an ischemic area).

The increase in each MCG parameter in Figure 4 and the abnormal area in Figure 5, according to the increase in lesion sites, indicates that the increase in lesion areas enhances the residual electrical components or degrades normal electrical activities. The subtraction method is, therefore, helpful for estimating the lesion area easily.

On the other hand, a stenosis site in the coronary arteries was not found in the CAM of the subtracted waveform of each patient. This may be because the occupied area of each coronary artery becomes complementary in the ventricular muscle ${ }^{22}$ and the individual variation of coronary-artery shape results in difficulties in identifying it.

\section{Detection of Abnormalities in CHD Patients}

Other groups have detected abnormalities in CHD patients by using magnetic field parameters (MFPs), which are calculated from the MCG data in the ventricular repolarization phase. ${ }^{10,11}$ In particular, it has been reported that the four MFPs are the most sensitive indicators for diagnosing CHD patients ${ }^{10}$ and the detection sensitivity (86.4\%) and specificity $(82.5 \%)$ possible by using the MFPs is higher than our results (sensitivity: $74.6 \%$; specificity: $84.1 \%$ ). However, quoting the same patients' data, our previous report clarified the decrease in MFP sensitivity and specificity compared with the results obtained with our current-distribution parameters (CDPs). ${ }^{16} \mathrm{We}$ consider that $\mathrm{CDP}$ is the most important parameter for detecting CHD. Although the sensitivity and specificity obtained with CDPs are also high, the correlation between abnormal score and lesion area is not good. In this study, we assumed that abnormal current distribution depending on CHD is extracted by subtraction of normal electrical-current activities, and 
we found a good correlation between the number of lesion sites and the magnitude of subtracted waveforms.

Furthermore, as for the subtraction MCG method, the highest values of MCG parameters of patients with MI areas, as shown in Figure 6, reflect the lack of electrical activation (depending on the infarction area). The difference between MI and ischemia, therefore, shows that ischemic status can be evaluated with this method. Consequently our proposed CHD-evaluation method using subtracted MCG ST-T waveforms makes it possible to estimate sizes of infarctions and coronary-artery lesions.

Acknowledgments: We are grateful to Shigeaki Naito, Toru Okihara, Hiroki Ihara, Masafumi Iso, and Hiroyuki Suzuki of HitachiHigh Technologies for planning the MCG measurements.

\section{LIMITATION OF THIS STUDY}

This study has the following limitations. First, CHD patients were identified in terms of coronary artery stenosis only; therefore, the status such as ischemia, MI, and stable/unstable angina of all patients were not distinguished. Second, the subtraction method may be insufficient in the case of MI patients with a low-amplitude $\mathrm{T}$ wave whose $\mathrm{T}$ wave peak cannot be identified because the subtraction method needs a correct T-wave peak as a reference point.

\section{REFERENCES}

1. Bluemke DA, Achenbach $S$, Budoff $M$, et al. Noninvasive coronary artery imaging (AHA Scientific Statement). Circulation 2008;118:586-606.

2. Delhaye D, Remy-Jardin M, Rozel C, et al. Coronary artery imaging during preoperative CT staging: Preliminary experience with 64-slice multidetector CT in 99 consecutive patients. Eur Radiol 2007:17:591-602.

3. Freeman J, Ashley EA, Froelicher V. Should the exercise ECG be used to screen for sudden cardiac death? Eur Heart J 2009;30:528-529.

4. Lin F, Shaw L, Berman D, et al. Multidetector computed tomography coronary artery plaque predictors of stressinduced myocardial ischemia by SPECT. Atherosclerosis 2008:197:700-709.

5. Kandori A, Ogata K, Watanabe Y, et al. Space-time database for standardization of adult magnetocardiogram- Making standard MCG parameters. Pacing Clin Electrophysiol 2008:31:422-431.
6. Kandori A, Ogata K, Miyashita T, et al. Standard template of adult magnetocardiogram. Ann Noninvasive Electrocardiol 2008; 13:390-399.

7. Tsukada $\mathrm{K}$, Miyashita T, Kandori A, et al. An iso-integral mapping technique using magneto-cardiogram, and its possible use for diagnosis of ischemic heart disease. Int J Card Imaging 2000;16:55-66.

8. Kandori A, Kanzaki H, Miyatake $\mathrm{K}$, et al. A method for detecting myocardial abnormality by using a current-ratio map calculated from an exercise-induced magnetocardiogram. Med Biol Eng Comput 2001;39:29-34.

9. Kanzaki H, Nakatani S, Kandori A, et al. A new screening method to diagnose coronary artery disease using multichannel magnetocardiogram and Simple Exercise. Basic Res Cardiol 2003:98:124-32.

10. Park JW, Hill PM, Chung N, et al. Magnetocardiography predicts coronary artery disease in patients with acute chest pain. Ann Noninvasive Electrocardiol 2005;10:312-323.

11. Tolstrup K, Madsen BE, Ruiz JA, et al. Non-invasive resting magnetocardiographic imaging for the rapid detection of ischemia in subjects presenting with chest pain. Cardiology 2006;106:270-276.

12. Yamada S, Yamaguchi I. Magnetocardiograms in clinical medicine: Unique information on cardiac ischemia, arrhythmias, and fetal diagnosis. Intern Med 2005;44:1-19.

13. Hailer B, Van Leeuwen P, Chaikovsky I, et al. The value of magnetocardiography in the course of coronary intervention. Ann Noninvasive Electrocardiol 2005;10:188-196.

14. Lim HK, Kim K, Lee YH, et al. Detection of non-STelevation myocardial infarction using magnetocardiogram: New information from spatiotemporal electrical activation map. Ann Med 2009;22:1-14.

15. Lim HK, Kwon H, Chung N, et al. Usefulness of magnetocardiogram to detect unstable angina pectoris and non-ST elevation myocardial infarction. Am J Cardiol 2009;103:448454.

16. Ogata K, Kandori A, Watanabe Y, et al. Repolarization spatial-time current abnormalities in patients with coronary heart disease. Pacing Clin Electrophysiol 2009;32:516-524.

17. Tsukada K, Kandori A, Miyashita T, et al. A simplified superconducting interference device system to analyze vector components of a cardiac magnetic field. Proceedings 20th Int. Conf., IEEE/EMBS, Hong Kong, 1998;524-527.

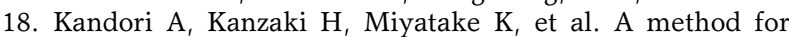
detecting myocardial abnormality by using a total currentvector calculated from ST-segment deviation of a magnetocardiogram signal. Med Biol Eng Comput 2001;39:21-28.

19. Shimizu W, Antzelevitch C. Differential effects of betaadrenergic agonists and antagonists in LQT1, LQT2 and LQT3 models of the long QT syndrome. J Am Coll Cardiol 2000;35:778-786.

20. Kandori A, Hosono T, Kanagawa T, et al. Detection of atrialflutter and atrial-fibrillation waveforms by fetal magnetocardiogram. Med Biol Eng Comput 2002;40:213-217.

21. Lemay M, Vesin JM, van Oosterom A, et al. Cancellation of ventricular activity in the ECG: Evaluation of novel and existing methods. IEEE Trans Biomed Eng 2007:54:542546.

22. Kiriyama $\mathrm{T}$, Hayashi $\mathrm{H}$, Kumita S. Myocardial perfusion imaging with single-photon emission computed tomography for ischemic heart disease. J Jpn Coll Angiol 2009;49:481486. 